\title{
Study of surfactant-liposome interactions at sublytic level by means of a surface probe
}

\author{
M. Cócera ${ }^{a}$, O. López ${ }^{\text {a }}$, J. Estelrich ${ }^{\mathrm{b}}$, J.L. Parra ${ }^{\mathrm{a}}$ and A. de la Maza ${ }^{\mathrm{a}, *}$ \\ ${ }^{a}$ Departamento de Tecnología de Tensioactivos, Instituto de Investigaciones Químicas y Ambientales \\ de Barcelona (I.I.Q.A.B.), Consejo Superior de Investigaciones Científicas (C.S.I.C.), C/ Jordi Girona, \\ 18-26, 08034 Barcelona, Spain \\ ${ }^{\mathrm{b}}$ Departament de Fisicoquímica, Facultat de Farmàcia, Universitat de Barcelona, Av. Joan XXIII s/n, \\ 08028 Barcelona, Spain
}

\begin{abstract}
The incorporation of the sodium lauryl ether sulfate (SLES) on phosphatidylcholine (PC) liposomes has been studied with time using the fluorescent probe 2-( $p$-toluidinyl)-naphthalene-6-sodium sulfonate (TNS). This probe reports changes on the surface potential of PC liposomes by effect of the anionic surfactant adsorption. The addition of small SLES amounts promoted an abrupt fall in the fluorescence intensity (F.I.) and this fall was already detected 10 secs after mixing. Only slight changes with time were observed in the F.I. of SLES-liposome-probe systems. These results indicate a fast and almost complete incorporation of SLES on the liposome surface. The surfactant/lipid molar ratios $(R e)$ and the surfactant partition coefficients bilayer/aqueous phase $(K)$ were determined from the linear dependence between lipid and surfactant concentrations obtained for a fixed number of surfactant molecules on the liposome surface. The Re values indicated that the highest SLES ability to adsorb on the surface occurs at the initial adsorption moments. The $K$ values indicated that the affinity of the surfactant by the liposomes decreased after about 7500 surfactant molecules were adsorbed on the bilayer. This fact is probably caused by the increase of electrostatic repulsion between surfactant monomers in the bulk solution and the bound surfactant. The aforementioned linear dependence obtained from the data for a given number of SLES molecules on the bilayer and the range of SLES concentrations used (below/above its critical micelle concentration, CMC) suggest an adsorption mechanism regardless of the surfactant concentration: a monomeric adsorption of SLES is always assumed in both above/below surfactant CMC. In comparison with the higher adsorption reported for the analogous sodium dodecyl sulfate, the ethylene oxide moles in SLES could hinder its incorporation on PC liposomes. Thus, this study of sublytic interactions allows to follow the incorporation of surfactants on the lipid bilayers and to compare the effect of different surfactants on the membranes before the lysis.
\end{abstract}

Keywords: Phosphatidylcholine liposomes, sodium dodecyl sulfate, sodium lauryl ether sulfate, sublytic interaction, fluorescent probe 2-( $p$-toluidinyl)-naphthalene-6-sodium sulfonate

\section{Abbreviations}

PC, phosphatidylcholine; SLES, sodium lauryl ether sulfate; SDS, sodium dodecyl sulfate; EO, ethylene oxide; TRIS, tris(hydroximethyl)-aminomethane; TNS, 2-(p-toluidinyl)-naphthalene-6-sodium sulfonate; $\Psi_{o}$, surface potential; $\sigma_{o}$, surface charge density; $S_{\text {ext }}$, outer vesicle surface; $S_{\mathrm{W}}$, the surfactant concentration in the aqueous medium; $R e$, effective surfactant to PC molar ratio; $K$, bilayer/aqueous phase surfactant partition coefficient; $r^{2}$, the regression coefficients; CMC, critical micellar concentration; DLS, dynamic light scattering; $\tau_{2}$, micellar relaxation time.

${ }^{*}$ Corresponding author. Tel.: +34 9340061 61; Fax: +34 932045904. 


\section{Introduction}

The anionic surfactants sodium dodecyl sulphate (SDS) and sodium lauryl ether sulphate (SLES) are widely used surfactants in industry, household and personal care products because of its good foaming properties. The structural difference between these molecules is the ethylene oxide (EO) units spacing the hydrophilic and the hydrophobic part in the SLES. This surfactant has demonstrated to be milder than SDS [1] on the skin. Hence, some authors reported that SDS had higher capacity of irritation in vivo than SLES (at $1 \%$ concentration) using soap chamber test [2]; others observed a decreased erythema with mixtures of SDS and SLES (30\%) applied in human volunteers against the use of 20\% SDS alone [3]. The addition of EO units promotes lower in vivo irritation and decreases the values in corneosurfametry [4]. Thus, the presence of EO moles appears to be the cause of the milder behaviour of the surfactants on the skin. In this sense, the use of phosphatidylcholine (PC) liposomes could help to understand the role of EO in the surfactant molecule and its relation with the irritation. Although we are aware that the PC liposomes are a simple membrane model, they can provide valuable information that would be difficult to attain in vivo.

In previous papers, we studied different aspects involving the interaction of SDS with phosphatidylcholine (PC) and stratum corneum lipid liposomes [5-7]. These works reported information about the solubilization process, as well as the initial mechanism of the surfactant incorporation on the liposomes. In the present work, we seek to extend these investigations by studying some kinetic and structural aspects of the adsorption of SLES with 2 EO units on PC bilayers. To this end, we used the probe 2-( $p$-toluidinyl)-naphthalene-6-sodium sulfonate (TNS), which reports on the surface potential $\left(\Psi_{o}\right)$ of membranes [8-10]. The surfactant adsorption is associated to the $\Psi_{o}$ changes of the liposome/probe systems as a function of the SLES concentration and of the incubation time. This simple spectroscopy technique has been useful to study how the EO units affect the SLES adsorption mechanism on the PC vesicles in relation to the data reported for SDS. Our investigations could help to clarify why the EO moles reduce the irritant activity of the SLES on the skin.

\section{Materials and methods}

Lipoid S100 as phosphatidylcholine (PC) from soybean lecithin was purchased from Lipoid GmbH (Ludwigshafen, Germany). Sodium lauryl ether sulfate (SLES) containing 2-ethylene oxide (EO) moles (27\%) and sodium dodecyl sulfate were supplied by Albright and Wilson Iberica (Barcelona, Spain) and Merck (Darmstadt, Germany), respectively. Tris(hydroximethyl)-aminomethane (TRIS) was obtained from Merck. TRIS buffer was prepared as $5.0 \mathrm{mM}$ TRIS buffer adjusted to $\mathrm{pH} 7.4$ with $\mathrm{HCl}$ and containing $100 \mathrm{mM} \mathrm{NaCl}$. The fluorescent agent 2-( $p$-toluidinyl)-naphthalene-6-sodium sulfonate (TNS) purchased from Sigma Chemical Co. (St. Louis, MO) was prepared as $100 \mu \mathrm{M}$ TNS in TRIS buffer and stored at $4^{\circ} \mathrm{C}$. Polycarbonate membranes of 800,400 , and $200 \mathrm{~nm}$ pore size were purchased from Nucleopore (Pleasanton, CA).

\subsection{Determinations of the critical micelle concentrations of the surfactants}

The surface tension of the surfactant solutions in $5 \mathrm{mM}$ TRIS buffer containing $100 \mathrm{mM} \mathrm{NaCl}$ adjusted to $\mathrm{pH} 7.4$ was measured at room temperature using a Wilhelmy plate method (Krüss K-12 Processor Tensiometer), which determines directly the real surface tension values at equilibrium. The surfactant critical micelle concentration (CMC) was determined from the abrupt change in the slope of the surface tension values versus surfactant concentration. 


\subsection{PC liposomes including TNS probe and interaction with SLES}

A PC film was obtained by rotary evaporation of the solvent from a chloroform containing PC solution. Unilamellar liposomes of a defined size (of about $200 \mathrm{~nm}$ ) were prepared by a 10-fold passage through 800-400-200 nm polycarbonate membranes [11] of vesicles previously obtained by hydration of the PC film with TRIS buffer. To incorporate the probe on the vesicle surface, TNS buffered system was added to liposomes. A period of about 30 minutes was needed to obtain a complete and stable incorporation of TNS on the liposome (measured as a constant F.I. value of the liposome/probe). Afterwards, equal volumes of appropriate surfactant solutions were added to the liposome/probe suspensions, and the fluorescence measurements were performed at $25^{\circ} \mathrm{C}$.

\subsection{Measurements of the fluorescence of TNS probe incorporated on different systems}

TNS is a probe of membrane surface potential $\left(\Psi_{o}\right)$ introduced by Eisenberg et al. [8]. The fluorescence of this probe is extremely low in water. The addition to liposomes led to the adsorption of these molecules on the bilayer surface increasing its fluorescence, which is quenched by anionic amphiphiles. Kachel et al. located TNS within the polar head group region of the phospholipids (at 16-18 $\mathrm{A}$ from the bilayer centre), i.e., with its charged sulfonic acid group anchored at a shallow location [9]. This probe has been used to determine the $\Psi_{o}$ of liposomes containing either anionic lipids [8,10], or surfactants $[12,13]$, and to study the self-association of bile salts in water [14]. Fluorescence measurements were performed on a spectrofluorometer (Shimadzu RF-540, Kyoto Japan) with excitation wavelength $\left(\lambda_{\text {exc }}\right)$ at $321 \mathrm{~nm}$ and emission $\left(\lambda_{\mathrm{em}}\right)$ at $446 \mathrm{~nm}$.

The $\Psi_{o}$ of vesicles was calculated from the ratio of fluorescence of liposomes and those containing surfactant at the same lipid concentration using the relation:

$$
f(-) / f(0)=\exp \left\{F \Psi_{\mathrm{o}} / R T\right\}
$$

where $f(0)$ and $f(-)$ are the fluorescence intensity in the absence and in the presence of quencher, $F$ is the Faraday constant, $R$ is the gas constant, and $T$ is the absolute temperature. This equation is useful when a small fraction of binding sites are occupied by TNS at the liposome surface, and when the aggregates provide very similar surface environments for TNS to give equal life times, as occurred in our experimental conditions. From the $\Psi_{o}$ values (expressed in volts), the surface charge density $\left(\sigma_{o}\right)$ expressed in $\mu \mathrm{C} . \mathrm{cm}^{-2}$ for symmetrical electrolytes may be calculated by means of [15]:

$$
\sigma_{o}=11.74 \sqrt{ } \mathrm{C} \cdot \sinh \left\{z e \Psi_{o} / 2 k T\right\}
$$

where $C$ is the electrolyte concentration in mol. $1^{-1}, z$ the valence of ions, $e$ the elementary charge, $k$ the Boltzmann constant and $T$ the absolute temperature. The number of charged molecules $(n)$ can be obtained from:

$$
n=\left(\sigma_{o} / 1.60219 \cdot 10^{-19}\right) \cdot S_{\mathrm{ext}} \cdot 10^{-22},
$$

where $S_{\text {ext }}$ is the external surface of a vesicle expressed in $\AA^{2}$. 


\subsection{Parameters involved in the interaction of SLES with PC liposomes}

In the analysis of the equilibrium partition model proposed by Schurtenberger et al. [16] for bile salt/lecithin systems, Lichtenberg et al. [17] and Almog et al. [18] have shown that for a mixing of lipids (at a conc. $L(\mathrm{mM})$ ) and surfactant (at a conc. $S_{\mathrm{T}}(\mathrm{mM})$ ), in dilute aqueous media, the distribution of surfactant between lipid bilayers and aqueous media obeys a partition coefficient $K$, given (in $\mathrm{mM}^{-1}$ ) by:

$$
K=R e / S_{\mathrm{W}}[1+R e]
$$

where $R e$ is the effective surfactant to lipid molar ratio in the bilayers and $S_{\mathrm{W}}$ is the surfactant concentration in the aqueous medium $(\mathrm{mM})$. The $R e, S_{\mathrm{W}}$ and $K$ parameters were determined on the basis of the linear dependence between the surfactant concentrations needed for the adsorption of a given number of surfactant molecules on the bilayer surface and the PC concentration, which is described by the equation:

$$
S_{\mathrm{T}}=S_{\mathrm{W}}+R e \cdot[\mathrm{PC}],
$$

where $S_{\mathrm{T}}$ is the total surfactant concentrations. The $R e$ and $S_{\mathrm{W}}$ are in each curve the slope and the ordinate at the origin (zero PC concentration), respectively. The $K$ parameters (bilayer/aqueous phase partition coefficient) were determined from the Eq. (4).

\section{Results and discussion}

Figure 1 shows the molecular structures for the surfactants (A), indicating the presence of EO units in the SLES, and for the probe (B). Both surfactants have the same hydrocarbon chain length and the same sulphate polar head group.

Figure 2 plots the surface tensions of surfactant solutions versus surfactant concentrations. The CMCs corresponding to SLES and SDS resulted $0.12 \mathrm{mM}$ and $0.75 \mathrm{mM}$, respectively, in the experimental conditions described above. The higher CMC for the SDS shows that the EO units in the SLES structure decrease its CMC, in line with the results reported by Vollhardt et al. [19]. These authors associated the introduction of EO units with an increase in the surface activity. However, this work did not consider the value of the surface tension at the CMC $\left(\gamma_{\mathrm{CMC}}\right)$, which has been used as one criteria of surface activity of the system by other authors (the lower the $\gamma_{\mathrm{CMC}}$, the higher the surface activity [20]). In our experiments, $\gamma_{\mathrm{CMC}}$ for SLES and SDS were 29.9 and $26.4 \mathrm{mN} / \mathrm{m}$, respectively. Hence, we may assume that the EO units in SLES decrease its surface activity.

The F.I. of the probe remained almost constant for a minimum of 6 hours after mixing liposomes with TNS, indicating no migration of the probe to the interior of vesicles under our experimental conditions.
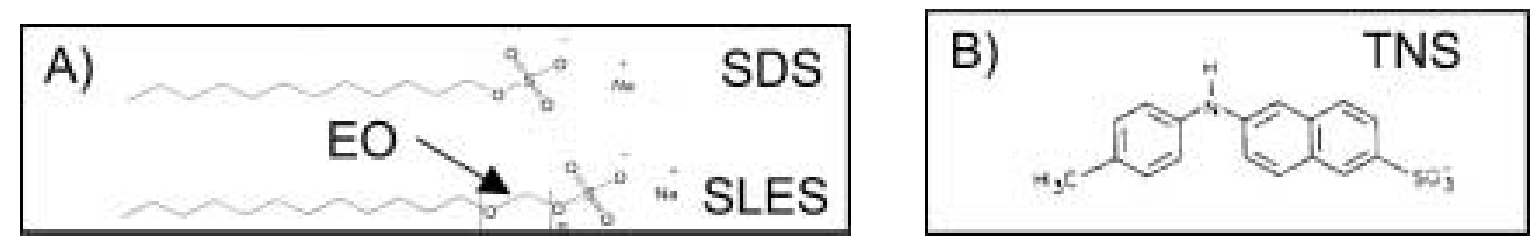

Fig. 1. Molecular structures of the surfactants (A) SLES and SDS, and the surface probe TNS (B). 


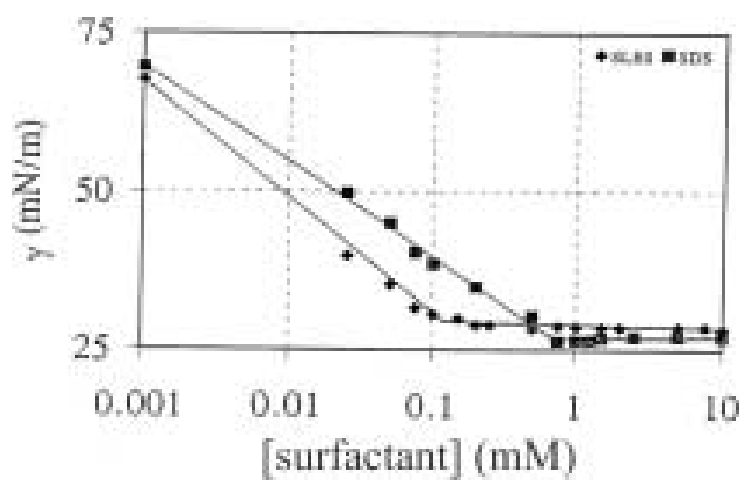

Fig. 2. Surface tensions of SLES and SDS solutions (in $5 \mathrm{mM}$ TRIS buffer containing $100 \mathrm{mM} \mathrm{NaCl}$ and adjusted to $\mathrm{pH} 7.4$ ) versus surfactant concentrations. The abrupt change in the slope of the surface tension values determines the surfactant critical micelle concentrations $(\mathrm{CMC})$. The measurements were performed at room temperature.

This fact is in line with the work of Eastman et al. [21], who reported the requirement of a transmembrane $\mathrm{pH}$ gradient to explain the movement of TNS into lipid monolayer. A molar ratio PC/TNS of 100 results in a F.I. proportional to the number of TNS molecules adsorbed on the bilayer, the probe fluorescence in aqueous solution being negligible with respect to the TNS bound to vesicles. The F.I. of the probe when is added to SLES containing-PC liposomes at the equilibrium (20 hours after mixing) is constant with time (data not show). This control experiments allows us to conclude that TNS does not translocate to the inner monolayer and, hence, the changes in the fluorescence emission described in this work are promoted by the surfactant adsorption on liposomes. The F.I. of liposome/probe solutions with increasing surfactant amounts after different incubation times was determined. The assays were carried out in triplicate and the final results are the averages. The standard deviations of the data for each point were lower than $1.1 \%$. In each curve, maximum (100\%) F.I. corresponds to liposomes/probe lacking surfactant after the same period of time.

To determine the $R e, S_{\mathrm{W}}$, and $K$ parameters for the adsorption of a given number of surfactant molecules on the liposome surface, we first measured the changes of the F.I. of the systems versus SLES concentration. The $\Psi_{o}$ calculated from these values by applying Eq. (1) allows to determine the changes in the surface charge density $\left(\sigma_{o}\right)$ of the liposomes (Eq. (2)) and, hence, the variation in the number of SLES molecules adsorbed per vesicle on the outer membrane leaflet (Eq. (3)). Unilamellar and spherical liposomes were used with a diameter of $190 \mathrm{~nm}$ [12]. In previous works, only slight changes in the liposome size (mixed vesicles) were determined by transmission electron microscopy and dynamic light scattering (DLS) at subsolubilizing concentrations of anionic surfactants [5,22]. Thus, we can assume that the liposome size remained almost constant throughout the experiment. Taking into account a surface area of $70 \AA^{2}$ for each lipid molecule, we obtained an outer vesicle surface of $11.3 \times 10^{6} \AA^{2}\left(S_{\text {ext }}\right.$, Eq. (3)).

The F.I. curves $10 \mathrm{sec}$ after mixing liposomes (PC concentration from 0.25 to $2.0 \mathrm{mM}$ ) with SLES are plotted in Fig. 3. The addition of a small amount of surfactant (lower than its CMC) always resulted in an abrupt decrease in the F.I. This initial decrease and the slight fluorescence changes with time (data not shown) indicate a fast and almost complete adsorption process, in line with our previous studies involving anionic surfactants and PC liposomes [6,13]. This rapid adsorption (initial step of the solubilization process) was also detected by DLS technique for the SDS [22]. The slight variations (increase) in the F.I. with time after SLES addition could be attributed to the inner leaflet migration of the surfactant. This phenomenon was described for the analogue SDS in a previous work [23]. Kragh-Hansen et al. 


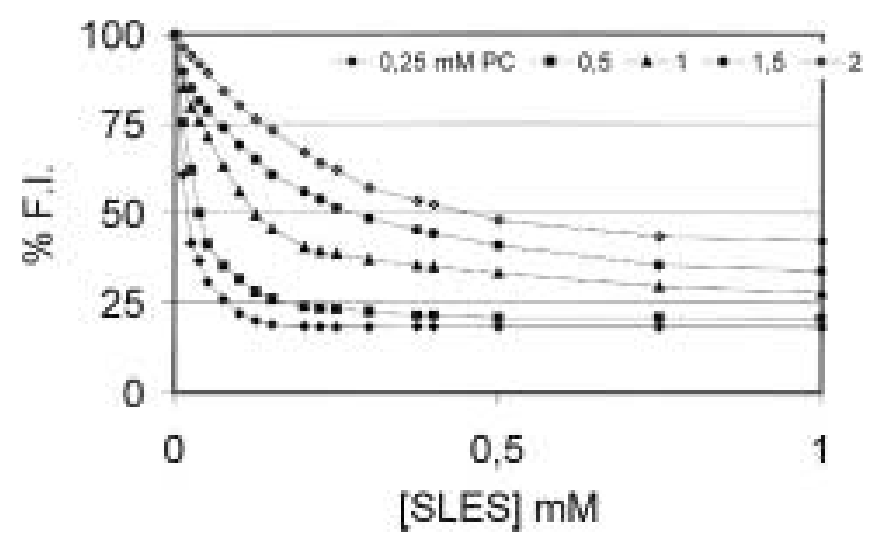

Fig. 3. Variation in the fluorescence intensity of PC bilayers (PC concentration from 0.25 to $2.0 \mathrm{mM}$ and PC/TNS molar ratio 100) containing increasing amounts of SLES after 10 seconds of incubation. In each curve fluorescence intensity (F.I.) $100 \%$ (maximum) corresponded to liposomes/probe without surfactant after the same period of time. Symbols: PC concentration: $0.25 \mathrm{mM}(\diamond), 0.5 \mathrm{mM}(\boldsymbol{\square}), 1.0 \mathrm{mM}(\boldsymbol{\Delta}), 1.5 \mathrm{mM}(\bullet)$, and $2.0 \mathrm{mM}(\diamond)$.

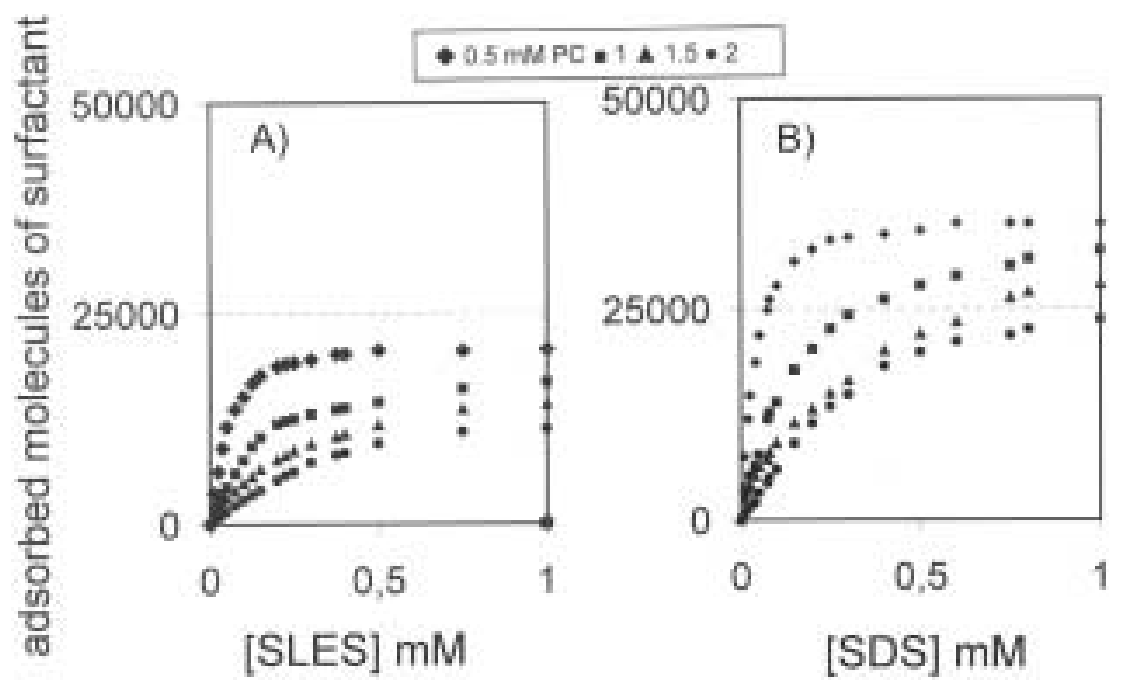

Fig. 4. Variation in the number of surfactant molecules adsorbed on the bilayer surface per vesicle (PC concentration from 0.5 to $2.0 \mathrm{mM}$ and PC/TNS molar ratio 100) versus the SLES concentration (A) and SDS concentration (B) in the systems after 10 seconds of incubation. Symbols: PC concentration: $0.5 \mathrm{mM}(\diamond), 1 \mathrm{mM}(\boldsymbol{\nabla}), 1.5 \mathrm{mM}(\boldsymbol{\Delta})$, and $2 \mathrm{mM}(\bullet)$.

demonstrated a slow flip-flop for the SDS because of the marked hydrophilic properties of its head group [24], which is very similar to that of the SLES.

Figure 4 plots the number of SLES (A) and SDS (B) molecules adsorbed per vesicle versus the added surfactant concentrations after $10 \mathrm{sec}$ of incubation. This figure shows that for the same PC and surfactant concentrations only half of SLES molecules were incorporated onto liposome surface. A similar effect occurred after different periods of incubation (results not shown). The presence of EO in SLES increases its overall size of the polar head group, thereby increasing the area occupied per surfactant molecule and possibly hindering an optimal packing on the interface. Hence, the EO units in the molecular structure of the SLES hinders its adsorption capacity in spite of the similar solubilizing ability reported for both surfactants [25]. In fact, it is commonly accepted that a potential irritant needs to penetrate through the 


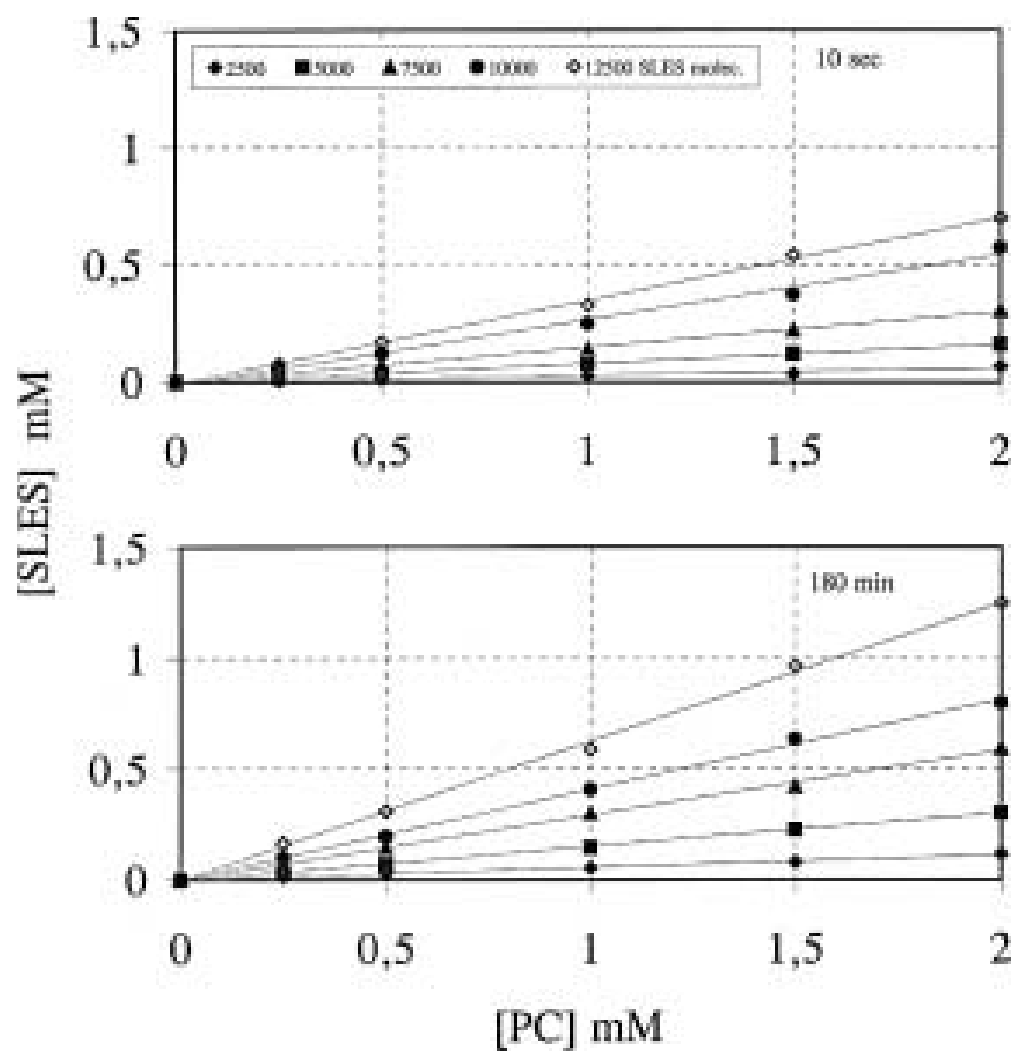

Fig. 5. Surfactant concentration resulting in different number of surfactant molecules incorporated in the outer surface of liposomes (PC concentration from 0.25 to $2.0 \mathrm{mM}$ and PC/TNS molar ratio 100) versus the liposome PC concentration, after 10 seconds, and 180 min of incubation. Symbols: number of SLES molecules incorporated: $2500(\checkmark), 5000(\mathbb{\square}), 7500(\mathbf{\Lambda})$, $10000(\bullet)$, and $12500(\diamond)$.

stratum corneum lipids before to exert its toxic effect on the skin. As a consequence, the milder effect on the skin reported for SLES [1-4] seems to be more related to this decreased adsorption than to its capacity to saturate or to solubilize liposomes.

The SLES concentrations needed to produce the adsorption of increasing number of surfactant molecules per vesicle (from 2500 to 12500 ) versus the PC concentration for $10 \mathrm{sec}$ and 180 minutes were plotted in Fig. 5. A linear relationship was established in each case (for different incubation periods). This linear correlation indicated a similar adsorption behaviour in the range of SLES and PC concentrations used. Given that the surfactant concentrations used were lower/higher than its CMC, we can assume that this adsorption was always monomeric even using SLES concentrations above its CMC. This adsorption could follow the mechanism proposed by Lasic [26], who reported the adsorption either through hydrophilic holes created by monomers on the PC polar heads or via formation short-lived complexes of surfactant-PC polar heads. The monomeric adsorption at SLES concentrations above its CMC implicates a breaking of the micellar structure into monomers previous to its incorporation on the bilayer surface. The kinetic of this process is governed by the micellar relaxation time $\left(\tau_{2}\right)$, which was determined by Patist et al. [27] for the SDS. These authors determined for $2.5 \mathrm{mM}$ of SDS $\tau_{2}$ values of $10^{-4} \mathrm{sec}$ and reported that the presence of increasing number of EO units in non-ionic surfactants reduced this period. 
Table 1

Surfactant concentrations in the aqueous medium $\left(S_{\mathrm{W}}\right)$, surfactant to lipid molar ratios $(R e)$, and partition coefficients $(K)$ for different number of surfactant molecules adsorbed on PC liposomes and for increasing periods of incubation surfactant/liposome.

\begin{tabular}{|c|c|c|c|c|c|c|c|c|c|c|c|c|}
\hline \multirow{2}{*}{$\begin{array}{l}\text { Absorbed } \\
\text { SLES } \\
\text { molec/ } \\
\text { liposome }\end{array}$} & \multicolumn{4}{|c|}{10 seconds incubation } & \multicolumn{4}{|c|}{30 minutes incubation } & \multicolumn{4}{|c|}{180 minutes incubation } \\
\hline & $\begin{array}{c}S_{\mathrm{W}} \\
(\mathrm{mM})\end{array}$ & $\begin{array}{c}R e \\
\text { mole/ } \\
\text { mole }\end{array}$ & $\begin{array}{c}K \\
\left(\mathrm{mM}^{-1}\right)\end{array}$ & $r^{2}$ & $\begin{array}{c}S_{\mathrm{W}} \\
(\mathrm{mM})\end{array}$ & $\begin{array}{c}R e \\
\text { mole/ } \\
\text { mole }\end{array}$ & $\begin{array}{c}K \\
\left(\mathrm{mM}^{-1}\right)\end{array}$ & $r^{2}$ & $\begin{array}{c}S_{\mathrm{W}} \\
(\mathrm{mM})\end{array}$ & $\begin{array}{c}R e \\
\text { mole/ } \\
\text { mole }\end{array}$ & $\begin{array}{c}K \\
\left(\mathrm{mM}^{-1}\right)\end{array}$ & $r^{2}$ \\
\hline 2500 & 0.003 & 0.031 & 10.02 & 0.942 & 0.003 & 0.033 & 10.65 & 0.997 & 0.004 & 0.056 & 13.26 & 0.997 \\
\hline 5000 & 0.003 & 0.082 & 25.26 & 0.997 & 0.003 & 0.085 & 26.11 & 0.999 & 0.005 & 0.152 & 26.39 & 0.999 \\
\hline 7500 & 0.004 & 0.152 & 32.99 & 0.999 & 0.004 & 0.161 & 34.67 & 0.999 & 0.006 & 0.294 & 37.87 & 0.998 \\
\hline 10000 & 0.015 & 0.279 & 14.54 & 0.990 & 0.016 & 0.310 & 14.79 & 0.998 & 0.018 & 0.409 & 16.13 & 0.998 \\
\hline 12000 & 0.03 & 0.355 & 8.73 & 0.998 & 0.04 & 0.428 & 7.49 & 0.999 & 0.04 & 0.628 & 9.64 & 0.998 \\
\hline
\end{tabular}

Thus, it is expected for SLES a lower $\tau_{2}$ than for SDS indicating that the micellar breaking would not be a rate-limiting step in the SLES adsorption on liposomes.

Taking into account the Eq. (5) and the data from Fig. 5, $R e, K$, and $S_{\mathrm{W}}$ were determined. These parameters for different number of surfactant molecules adsorbed and various incubation times, together with the regression coefficients $\left(r^{2}\right)$ for each straight line are given in Table 1.

A very low free surfactant concentration $\left(S_{\mathrm{W}}\right)$ was detected even $10 \mathrm{sec}$ after incubation. This fact confirms the fast kinetic of the adsorption process, in which most of the surfactant molecules were incorporated on the vesicle surface after this short period of time. This finding is related to that reported for the kinetics of liposome solubilization by SDS using a DLS technique [22], in which no free surfactant micelles were detected in periods higher than $30 \mathrm{sec}$ of incubation.

As for the $R e$ values, the increase in the incubation time of liposome/probe-surfactant led to a progressive rise in these values. Given that the ability of the SLES molecules to be incorporated on the liposome surface is inversely related to the $R e$ value we may assume that the maximum ability always occurred $10 \mathrm{sec}$ after incubation, corroborating a very fast adsorption kinetics. Comparison of these $R e$ values with those reported for the SDS [6] for the same number of molecules incorporated shows that the SLES exhibits always higher values. This fact indicates the relative lower incorporation ability of the SLES, in line with the results discussed in Fig. 2 and with its aforementioned lower surface activity $\left(\gamma_{\mathrm{CMC}}\right)$.

The partition coefficient variations show that up to 7500 molecules of SLES adsorbed the $K$ increased (see Table 1); the adsorption of more molecules led to a decrease in $K$. This indicates a loss in the surfactant affinity with the vesicles possibly due to the electrostatic repulsion between the free and the bound surfactant monomers, which would hinder the incorporation of new monomers on the charged surface of liposomes. Comparing with the $K$ values reported for the SDS [6], for the same number of adsorbed molecules and for the same periods of incubation, we observed that the SLES had always higher values. This increased $K$ values for the SLES could be attributed to the EO units in its molecular structure, which lend to the surfactant molecule a more hydrophilic character.

\section{Conclusion}

From the data concerning to the SLES solutions, we see that the EO units in SLES decrease its CMC and its surface activity. The increase of the overall size of the polar head group of SLES seems difficult the optimal packing of the molecules in the interface. 
The decrease in the fluorescence promoted by small amounts of surfactant at short periods of time and the slight fluorescence changes with time indicate a fast and almost complete adsorption process, in which most of the surfactant molecules are incorporated on the vesicle surface. A slower surfactant migration to the inner leaflet of the bilayer seems occur, although more experimental evidences are needed to confirm this fact.

The $R e$ and $K$ parameters for the SLES at sublytic level are more unfavourable that those reported for the SDS indicating a lower capacity to be incorporated on the bilayer than the SDS. The higher molecular size and hydrophilic character of the SLES can hinder its adsorption on membranes and this could be the reason to explain the milder effect on the skin surface than that promoted by the SDS. Furthermore, a monomer adsorption seems a more realistic mechanism of interaction with the bilayers than a micellar adsorption.

In the present work we have demonstrated the suitability of this spectroscopy technique to study the adsorption of SLES on PC liposomes. The analysis of the physico-chemical parameters described here leads to very detailed results about the adsorption of this EO-containing surfactant. Despite of the complexity of these data, a simple and general aspect should be underlined: the important role of the EO units on surfactants properties. For the SLES, these properties seem to be more related to the adsorption than to the solubilizing ability of the surfactant. Hence, the milder effect on the skin reported for SLES would be associated with its decreased adsorption. These perspectives should be considered in future works related to the design and application of new topical compounds.

\section{Acknowledgements}

The authors thank ORTEVE (Barcelona, Spain) for the provision of the PC (Lipoid S100). This work was supported by funds from C.I.C.Y.T., Prog No 95-0340-OP, Spain.

\section{References}

[1] V. Charbonnier, B.M. Morrison, Jr., M. Paye and H.I. Maibach, Subclinical, non-erythematous irritation with an open assay model (washing): sodium lauryl sulfate (SLS) versus sodium laureth sulfate (SLES), Food Chem. Toxicol. 39 (2001), 279-286.

[2] H.C. Korting, T. Herzinger, A. Hartinger, M. Kerscher, T. Angerpointner and H.I. Maibach, Discrimination of the irritancy potential of surfactants in vitro by two cytotoxicity assays using normal human keratinocytes, HaCaT cells and 3T3 mouse fibroblasts: correlation with in vivo data from soap chamber assay, J. Dermatol. Sci. 7 (1994), 119-129.

[3] A. Dillarstone and M. Paye, Antagonism in concentrated surfactant systems, Contact Dermatitis 28 (1993), 198.

[4] V. Goffin, M. Paye and G.E. Piérard, Comparison of in vitro predictive test for irritation induced by anionic surfactants, Contact Dermatitis 33 (1995), 38-41.

[5] O. López, M. Cócera, E. Wehrli, J.L. Parra and A. de la Maza, Solubilization of liposomes by sodium dodecyl sulfate: new mechanism based on the direct formation of mixed micelles, Arch. Biochem. Biophys. 367(2) (1999), 153-160.

[6] M. Cócera, O. López, J. Estelrich, J.L. Parra and A. de la Maza, Kinetic and structural aspects of the adsorption of sodium dodecyl sulfate on phosphatidylcholine liposomes, Langmuir 16(9) (1999), 4068-4071.

[7] O. López, M. Cócera, A. de la Maza, L. Coderch and J.L. Parra, Different stratum corneum lipid liposomes as models to evaluate the effect of the sodium dodecyl sulfate, Biochim. Biophys. Acta 1508 (2000), 196-209.

[8] M. Eisenberg, T. Gresalfi, T. Riccio and S. McLaughlin, Adsorption of monovalent cations to bilayer membranes containing negative phospholipids, Biochemistry 18(23) (1979), 5213-5223.

[9] K. Kachel, E. Asunción-Puzalán and E. London, The location of fluorescence probes with charged groups in model membranes, Biochim. Biophys. Acta 1374 (1998), 63-76.

[10] M.C. Woodle, L.R. Collins, E. Sponsler, N. Kossovsky, D. Papahadjopoulos and F.J. Martin, Sterically stabilized liposomes. Reduction in electrophoretic mobility but not electrostatic surface potential, Biophys. J. 61 (1992), 902-910. 
[11] V. Dorovska-Taran, R. Wich and P. Walde, A 1H nuclear magnetic resonance method for investigating phospholipase D-catalyzed hydrolysis of phosphatidylcholine in liposomes, Anal. Biochem. 240 (1996), 37-47.

[12] M. Cócera, O. López, A. de la Maza, J.L. Parra and J. Estelrich, Electrokinetic study of the sublytic interaction of alkyl sulfates with phosphatidylcholine liposomes, Langmuir 15(6) (1999), 2230-2233.

[13] M. Cócera, O. López, J. Estelrich, J.L. Parra and A. de la Maza, Use of a fluorescence spectroscopy technique to study the adsorption of sodium dodecylsulfonate on liposomes, Chem. Phys. Lipids 109(20) (2001), 29-36.

[14] P. Mukerjee, Y. Moroi, M. Murata and A.Y.S. Yang, Bile salts as atypical surfactants and solubilizers, Hepatology 4 (1984), 61S-65S.

[15] J.R. Hunter, Zeta Potential in Colloid Science, Academic Press, London, 1981, p. 28.

[16] P. Schurtenberger, N. Mazer and W. Känzig, Micelle to vesicle transition in aqueous solution of bile salt and lecithin, J. Phys. Chem. 89 (1985), 1042-1049.

[17] D. Lichtenberg, R.J. Robson and E.A. Dennis, Solubilization of phospholipids by detergents. Structural and kinetic aspects, Biochim. Biophys. Acta 737(2) (1983), 285-304.

[18] S. Almog, B.J. Litman, W. Wimley, J. Cohen, E.J. Wachtel, Y. Barenholz, A. Ben Shaul and D. Lichtenberg, States of aggregation and phase transformations in mixtures of phosphatidylcholine and octyl glucoside, Biochemistry 29(19) (1990), 4582-4592.

[19] D. Vollhardt, G. Czichocki and R. Rudert, Effect of the molecular structure on the adsorption of alkyl ether sulphates and alkane ether sulphonates at the air-water interface, Colloids Surf. A 142 (1998), 315-322.

[20] M.J. Rosen, J. Am. Oil Chem. Soc. 51 (1974), 461-465.

[21] S.J. Eastman, M.J. Hope and P.R. Cullis, Transbilayer transport of phosphatidic acid in response to transmembrane $\mathrm{pH}$ gradients, Biochemistry 30(7) (1991), 1740-1745.

[22] O. López, M. Cócera, R. Pons, N. Azemar and A. de la Maza, Kinetic studies of liposome solubilization by sodium dodecyl sulfate based on a dynamic light scattering technique, Langmuir 14 (1998), 4671-4674.

[23] M. Cócera, O. López, J. Estelrich, J.L. Parra and A. de la Maza, Transbilayer movement of sodium dodecyl sulfate in large unilamellar phospholipid vesicles, Langmuir 15(20) (1999), 6609-6612.

[24] U. Kragh-Hansen, M. le Maire and J.V. Møller, The mechanism of detergent solubilization of liposomes and proteincontaining membranes, Biophys. J. 75 (1998), 2932-2946.

[25] A. de la Maza, L. Coderch, O. López, J. Baucells and J.L. Parra, Permeability changes caused by surfactants in liposomes that model the stratum corneum lipid composition, J. Am. Oil Chem. Soc. 74(7) (1997), 1-8.

[26] D.D. Lasic, Liposomes: from Physics to Applications, Elsevier Science Publishers, Amsterdam, 1993, Chapter 2.

[27] A. Patist, S.G. Oh, R. Leung and D.O. Shah, Kinetics of micellization: its significance to technological processes, Colloids Surf. A 176 (2001), 3-16. 


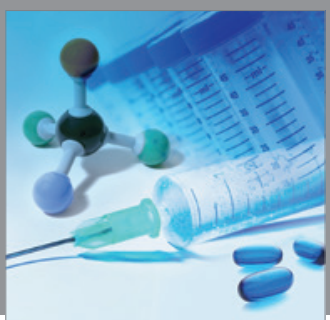

International Journal of

Medicinal Chemistry

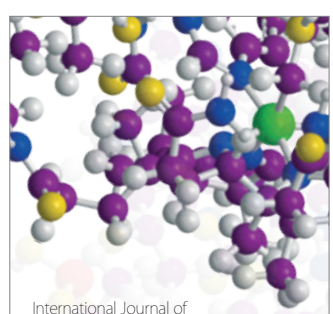

Carbohydrate Chemistry

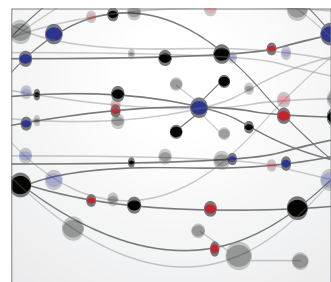

The Scientific World Journal
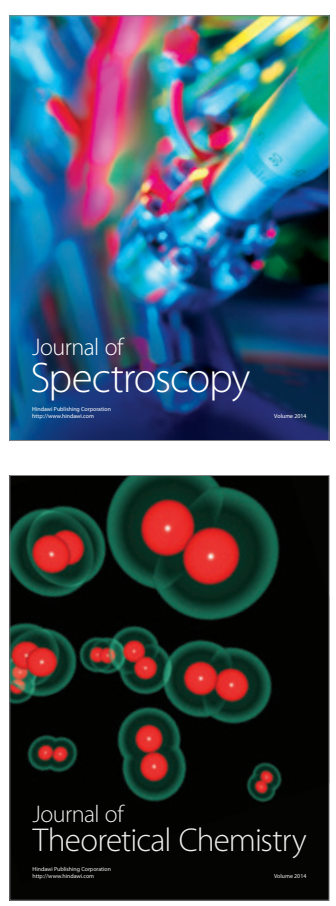
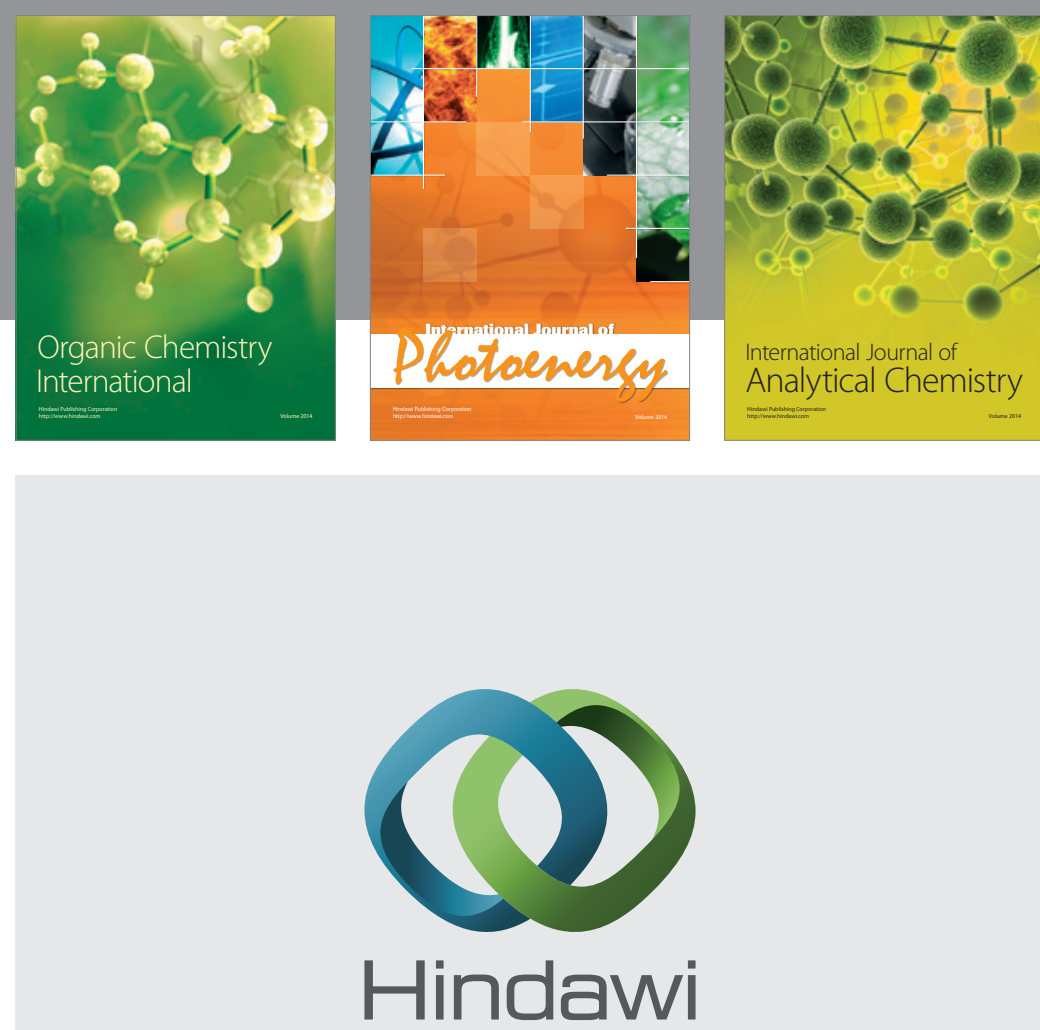

Submit your manuscripts at

http://www.hindawi.com
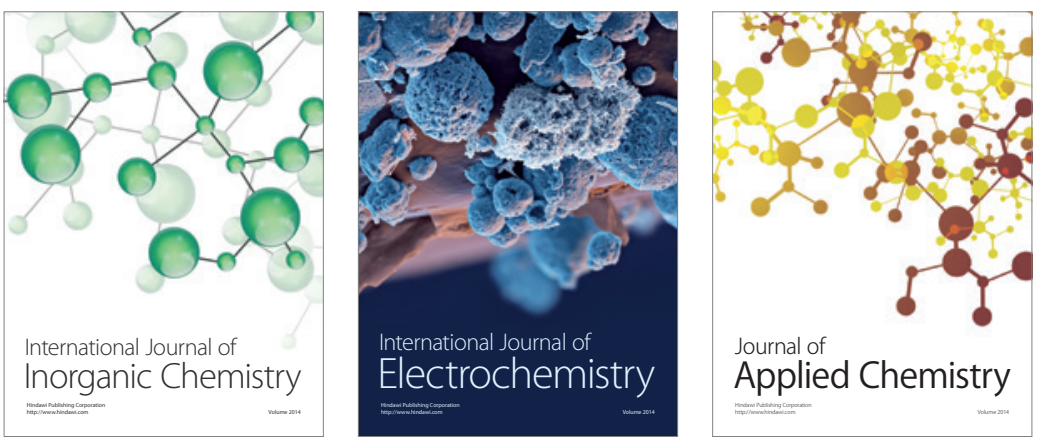

Journal of

Applied Chemistry
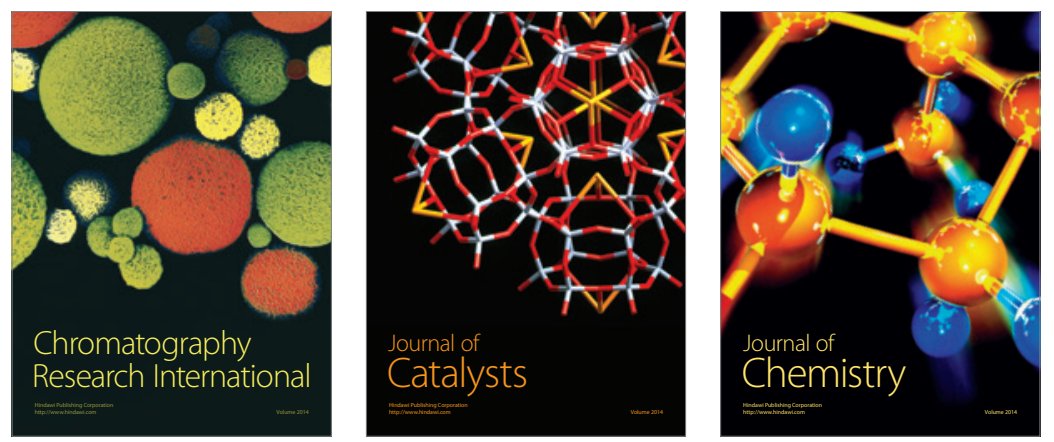
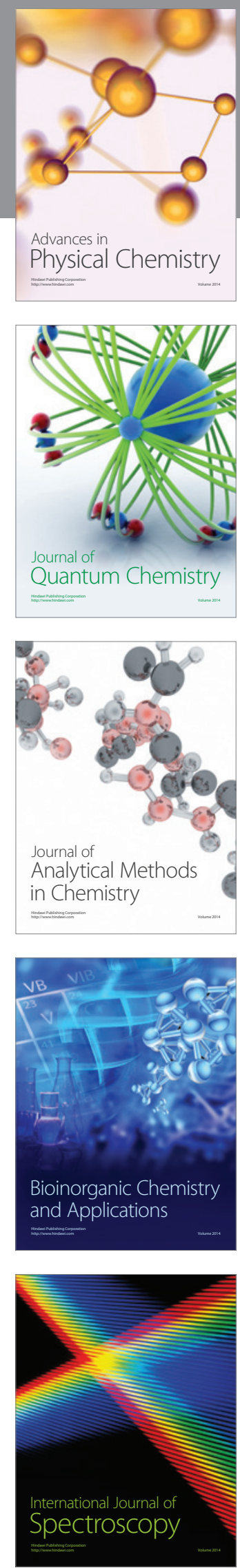